\title{
Simultaneous determination of zeatin and systemin by coupling graphene oxide-protected aptamers with catalytic recycling of DNase I
}

Chen Su, ${ }^{\mathrm{a}}$ Chen Liu, ${ }^{\mathrm{a}}$ Jinyang Chen, ${ }^{\mathrm{a}}$ Zilin $\mathrm{Chen}^{\mathrm{b}}$ and Zhike $\mathrm{He}^{* \mathrm{a}}$

${ }^{a}$ Key Laboratory of Analytical Chemistry for Biology and Medicine (Ministry of Education), College of Chemistry and Molecular Sciences, Wuhan University, Wuhan 430072, P. R. China. E-mail: zhkhe@whu.edu.cn; Fax:+86-27-6875-4067; Tel: +86-27-6875-6557

${ }^{b}$ School of Pharmaceutical Sciences, Wuhan University, Wuhan, 430071, China 


\begin{abstract}
Plant hormones are a collection of small molecules which play important roles in regulating the growth and development processes of plants. In this work, a sensitive and novel sensing platform for simultaneous multiplex detection of plant hormones is presented with choosing systemin and zeatin as the model targets. In the absence of the targets, the dye-labeled aptamers were adsorbed on the surface of graphene oxide (GO), result in the fluorescences of the dyes intensively quenched by GO which could also protect the aptamers from nuclease cleavage. While in the presence of the targets, the aptamers could be detached from the GO surface by specifically binding with targets. The fluorescences of the dyes were synchronously recovered and could be further amplified by DNase I catalytic recycling of self-produced reactants. So the two plant hormones can be simultaneously quantitatively determined by using synchronous scanning fluorescence spectrometry with no cross reaction between the two probes. The experimental results reveal that fluorescence intensities of two dyes exhibit good linear dependence on their target concentrations in the range of 0.67-6.0 $\mu \mathrm{M}$ and $0.33-5.0$ $\mu \mathrm{M}$ with the detection limit of $60 \mathrm{nM}$ and $35 \mathrm{nM}$ for zeatin and systemin, respectively.
\end{abstract}

Keywords: Plant hormones; Aptamer; Simultaneous determination; Signal amplification; DNase I 


\section{Introduction}

Plant hormones are a structurally unrelated collection of small molecules that play a crucial role in regulating numerous aspects of plant growth, development, and response to a wide range of biotic and abiotic stresses[1]. On the basis of their diverse structures and physiological functions, plant hormones are categorized into several major classes including auxin (AUX), gibberellin (GA), abscisic acid (ABA), cytokinin (CK), salicylic acid (SA), ethylene (ET), jasmonate (JA), brassinosteroid (BR), nitric oxide (NO), strigolactone (SL), and polypeptide hormone, and it is likely that additional plant hormones are yet to be discovered[2-4]. Enormous studies had revealed the role of each class of hormone, but in recent years, molecular genetic studies have been elucidating complicated crosstalk among the plant hormones[5-7]. Therefore, the development of accurate identification and simultaneous quantitative determination of multiple classes of plant hormones would be very meaningful in plant researches and applications.

With the development of analytical techniques, more accurate and sensitive determination methods for plant hormones come into being, including immunoassays, electroanalysis, and especially chromatographic methods, such as high-performance liquid chromatography (HPLC), gas chromatography (GC), and capillary electrophoresis (CE) coupled with different detectors[8-11]. While these methods have unique advantages of high sensitivity, fast speed, efficient separation, and simultaneous identification of multiple plant hormones, they are limited to the expensive devices and complicated coupling operations.

Aptamers are short single-stranded oligonucleotides that selectively recognize a wide range of targets, from small organic/inorganic molecules to proteins[12-14]. Recently, panels of aptamers have been selected for two plant hormones, systemin[15] and zeatin[16]. Moreover, the aptamers have been applied to the detections of systemin and zeatin, respectively. Based on these works, aptamer-functionalized $\mathrm{Zn}^{2+}$ doped CdTe QDs have been synthesized and applied to systemin detection by our group[17]. However, the affinity between plant hormones and their aptamers are not strong enough, and each aptamer binds to only a single target molecule. These weak affinity and 1:1 binding ratio limits signal enhancement as well as the sensitivity of the assay.

To overcome these problems, combining GO-protected DNA probes and cyclic enzymatic amplification 
method (CEAM)[18], we developed a new platform for simple, sensitive and multiplex analysis of plant hormones. DNase I is an endonuclease that nonspecifically cleaves DNA and releases di-, tri- and oligonucleotide products[19]. GO, a new kind of carbon nanomaterial, has been utilized for sensitive and selective detection of DNA[20,21] and proteins[22]. There are several advantages of GO-based fluorescent sensor[23-25]. First, GO has plenty of hydrophobic basal plane, which allow it to adsorb a wide range of biomolecules, such as Single-stranded DNA (ssDNA) on the surface via hydrophobic and p-stacking interactions[26, 27]. Second, GO has been reported to be a super-quencher to various kinds of fluorophores, via fluorescence resonance energy transfer (FRET) or non-radiative dipole-dipole coupling. Third, GO can protect aptamers from nuclease cleavage due to the steric-hindrance effects[28-31]. Fourth, GO, as a nanocarrier, has ultra-high surface areas for loading, including multiple molecules for multiplex sensing. These characteristics of GO provide technological base for this novel sensing platform.

In this work, GO strongly bind aptamers and protect them from nuclease cleavage, accompanied with the quenching of fluorescence of fluorophores conjugated with aptamers. However, when binds with the plant hormone, the aptamer is in a stable state with the rigid structure and thus released from the substrate of GO. Furthermore, the free aptamer can be cleaved by nuclease, liberates the fluorophore and ultimately releases the plant hormone. Then, the released plant hormone binds to another aptamer on GO to initiate a next round of cleavage, which leads to significant amplification of the signal. Because of the cyclic use of plant hormone, theoretically one plant hormone can initiate numerous probe digestions, which leads to a highly sensitive detection of plant hormone. Since the wavelength intervals between the maximum excitation wavelength and maximum emission wavelength of FAM and ROX are very close, the fluorescence signals of two fluorophores can be obtained simultaneously by using synchronous scanning fluorescence spectrometry. Thus, the simultaneous detection of two plant hormones can be realized by measuring fluorescence intensities of FAM and ROX, respectively. Compared with the other GO-based fluorescent sensor, the emergence of nuclease cleavage provides exciting new possibilities for achieving high sensitivity. What is more, the GO-protected multiplexed sensing strategy is firstly applied in the detection of plant hormones as we know. 


\section{Materials and methods}

\subsection{Apparatus and chemicals}

DNA sequences were synthesized by Shanghai Sangon Biotechnology Co., Ltd (Shanghai, China) and shown in the following:

1) Systemin probe: 5'-ROX-CTAACGGGTTTCGGGGGGGTAGGGAGGTTAG-3';

2) Zeatin probe: 5'-FAM-CGGATATGGTTAGGCAGGCATAAGAGGTTTATCCG-3'.

Peptide sequences were synthesized by ChinaPeptides Co., Ltd (Shanghai, China) and shown in the following:

1) Systemin: Ala-Val-Gln-Ser-Lys-Pro-Pro-Ser-Lys-Arg-Asp-Pro-Pro-Lys-Met-Gln-Thr-Asp;

2) Peptide 1: Cys-Cys-Val-Trp-Thr-Cys-Asp-Lys-Gly;

3) Peptide 2: Val-Val-Val-Thr.

All DNA samples were dissolved in $10 \mathrm{mM}$ Tris- $\mathrm{HCl}$ buffer solution $(\mathrm{pH} 7.4,150 \mathrm{mM} \mathrm{NaCl}, 10 \mathrm{mM}$ $\left.\mathrm{MgCl}_{2}, 10 \mathrm{mM} \mathrm{KCl}\right)$ and stored at $4{ }^{\circ} \mathrm{C}$ for use.

DNase I was obtained from Takara Biotechnology (Dalian) Co., Ltd. (China). GO was purchased from Sinocarbon Materials Technology Co., Ltd. Adenosine triphosphate (ATP) and trans-Zeatin were purchased from Sigma-Aldrich (USA). Dihydrozeatin (DHZ) was obtained from Olchemim (Czech Republic). All of the other reagents were of analytical-reagent grade or better, and the water used to prepare buffer solutions was prepared by a Milli-Q water system (Millipore Corp., USA) with resistivity of $18.2 \mathrm{MV} \mathrm{cm}$.

Fluorescence spectra were obtained in RF-5301PC spectrophotometer (Shimadzu, Japan), with $10 \mathrm{~nm}$ band-pass spectrometer slits. The fixed wavelength difference $(\Delta \lambda)$ of synchronous scanning fluorescence spectroscopy was set at $22 \mathrm{~nm}$.

2.2 Fluorescence response of 1:1 aptamer based assay towards zeatin and systemin. 
Systemin probe and Zeatin probe were diluted to $5.0 \mathrm{nM}$ and $4.0 \mathrm{nM}$ respectively in Tris- $\mathrm{HCl}$ buffer (50 $\mathrm{mM}, \mathrm{pH} 7.4,150 \mathrm{mM} \mathrm{NaCl}, 10 \mathrm{mM} \mathrm{MgCl} 2,10 \mathrm{mM} \mathrm{KCl}) .10 \mu \mathrm{L}$ of $0.1 \mathrm{mg} \mathrm{mL}^{-1} \mathrm{GO}$ suspension was added to the working solution and allowed to incubate for $10 \mathrm{~min}$ at room temperature. Afterwards, 4-(1-pyrenyl) butyric acid (PBA) $(3 \mu \mathrm{L}, 100 \mu \mathrm{M})$ was added to the mixture solutions as a surface-blocking agent. Then, different amounts of zeatin and systemin were added into the GO-aptamer solution and incubated for 2 hours. The final concentration of zeatin ranged from $5 \mu \mathrm{M}$ to $50 \mu \mathrm{M}$, and the final concentration of systemin ranged from $3.3 \mu \mathrm{M}$ to $30 \mu \mathrm{M}$. Finally the fluorescence intensities were detected.

\subsection{Fluorescence response of amplified aptamer based assay towards zeatin and systemin.}

Systemin probe and Zeatin probe were diluted to $5.0 \mathrm{nM}$ and $4.0 \mathrm{nM}$ respectively in Tris- $\mathrm{HCl}$ buffer (50 $\left.\mathrm{mM}, \mathrm{pH} 7.4,150 \mathrm{mM} \mathrm{NaCl}, 10 \mathrm{mM} \mathrm{MgCl}_{2}, 10 \mathrm{mM} \mathrm{KCl}\right) .10 \mu \mathrm{L}$ of $0.1 \mathrm{mg} \mathrm{mL}^{-1} \mathrm{GO}$ suspension was added to the working solution and allowed to incubate for $10 \mathrm{~min}$ at room temperature. Afterwards, 4-(1-pyrenyl) butyric acid (PBA) $(3 \mu \mathrm{L}, 100 \mu \mathrm{M})$ was added to the mixture solutions as a surface-blocking agent. Then, DNase I (0.5 U) and different amounts of zeatin and systemin were added to the GO-aptamer solution and incubated for 2 hours. The final concentration of zeatin ranged from $0.67 \mu \mathrm{M}$ to $6.0 \mu \mathrm{M}$, and the final concentration of systemin ranged from $0.33 \mu \mathrm{M}$ to $5.0 \mu \mathrm{M}$. Finally the fluorescence intensities were detected.

\section{Results and discussion}

\subsection{The principle of detection}

The principle of cyclic enzymatic amplification method for simultaneous detection of zeatin and systemin based on graphene oxide protected and quenched aptamers is depicted in Fig. 1. In the absence of targets, both

the probes of zeatin and systemin were adsorbed on the surface of GO via hydrophobic and p-stacking interactions between the nucleobases and GO. The interactions protected aptamers from nuclease cleavage. Furthermore, GO quenched the fluorescence of fluorophores conjugated with aptamers resulted from the efficient electronic transference of GO. When bound with the targets, the two kinds of probes were in a stable state with the rigid structures and released from the GO substrate. Whereupon the nuclease cleaved the released aptamers, liberated the fluorophores and ultimately released the targets, thereby, recovering the 
fluorescence of FAM and ROX. The released targets then bound with another aptamers, and a new cycle starts, which leaded to significant amplification of the signal. In this strategy, the fluorescence signals of FAM and ROX can be obtained simultaneously by using synchronous scanning fluorescence spectrometry, as the intervals between the maximum excitation wavelength and maximum emission wavelength of FAM and ROX are close between $23 \mathrm{~nm}$ and $20 \mathrm{~nm}$, respectively. Thus, the simultaneous detections of zeatin and systemin can be realized by simultaneously measuring the fluorescence signals of FAM and ROX.

\subsection{The feasibility of analytical method}

To demonstrate the feasibility of the simultaneous determination of two plant hormones, the feasible experiment had been carried out. From the results shown in Fig. 2, we could see two weak fluorescent emission (curve a) in the absence of targets due to the fluorescence quenching of FAM and ROX by GO. In the presence of zeatin and systemin, Systemin probe and Zeatin probe bound with their targets and were released from the GO substrate, the fluorescence signals of FAM and ROX were all enhanced synchronously (curve b). We then added DNase I in the above solution to evaluate the feasibility of the amplification. The cleavage of some of the aptamers adsorbed on GO by DNase I led to an increase in the background fluorescence in concert with the observed increase in the signal (curve c). Even so, the amplification strategy based on GO-protected aptamers led to a dramatic increase in the final fluorescence intensity of FAM and ROX upon the addition of zeatin and systemin (curve d). It was obvious that the changes in fluorescence signals after the addition of targets were significantly enhanced by using our amplification strategy over the 1:1 binding strategy.

\subsection{Effect of the concentration of $G O$}

The concentration of GO influences the fluorescence quenching efficiency of free probes. Therefore, the concentration of GO was investigated in this study. The results show that the fluorescence intensities decreased

quickly with the increased concentration of GO in the range of 1.0-5.0 $\mu \mathrm{g} \mathrm{mL}^{-1}$ and then tended to level off (Fig. 3). Therefore, $5.0 \mu \mathrm{g} \mathrm{mL}^{-1}$ of GO was selected for the following experiments.

\subsection{The simultaneous detection of the zeatin and systemin}

First, we characterized the simultaneous detection ability of GO-based aptamers by the 1:1 binding 
strategy. The fluorescence emission spectra of the multiplexed probes upon the addition of different concentration of targets were recorded by synchronous scanning fluorescence spectrometry. From the results shown in Fig. 4A, we could see the gradual increase of fluorescence intensity of two fluorophores, with increasing concentrations of zeatin and systemin. As show in the inserts of Fig. 4B and C, two good linear relationships between the fluorescence change $\left(F-F_{0}\right) / F_{0}$ and the concentrations of zeatin and systemin were obtained, respectively. For zeatin, the linear relationship was observed in the range from $5.0 \mu \mathrm{M}$ to $25 \mu \mathrm{M}$. The linear equation was $\mathrm{y}_{1}=0.01928 \mathrm{x}_{1}-0.00216\left(\mathrm{R}^{2}=0.99759\right)$ with a LOD as low as $1.3 \mu \mathrm{M}(3 \mathrm{~s}, \mathrm{n}=11)$. And for systemin, the linear relationship was observed in the range from $3.3 \mu \mathrm{M}$ to $13.3 \mu \mathrm{M}$. The linear equation was $\mathrm{y}_{2}=0.04913 \mathrm{x}_{2}+0.09684\left(\mathrm{R}^{2}=0.99579\right)$ with a LOD as low as $1.0 \mu \mathrm{M}(3 \mathrm{~s}, \mathrm{n}=11)$. The results reveal that the sensitivities of zeatin and systemin detection based on the strategy with no signal amplification were only limited in $\mu \mathrm{M}$ level.

In order to lower the LOD and improve the sensitivity of plant hormones detection, a signal amplification approach is needed. After simultaneously added targets and DNase I $(0.5 \mathrm{U})$, the subsequent fluorescence measurements showed a dramatic increase of fluorescence intensity of the two fluorophores with increasing concentrations of zeatin and systemin (Fig. 5A). As show in the inserts of Fig. 5B and C, two good linear relationship between the fluorescence changes $\left(F-F_{0}\right) / F_{0}$ and concentrations of zeatin and systemin were obtained, respectively. For zeatin, the linear relationship was observed in the range from $0.67 \mu \mathrm{M}$ to $3.0 \mu \mathrm{M}$. The linear equation was $\mathrm{y}_{1}=0.69603 \mathrm{x}_{1}-0.14873\left(\mathrm{R}^{2}=0.98552\right)$ with a LOD as low as $60 \mathrm{nM}(3 \mathrm{~s}, \mathrm{n}=11)$. And for systemin, the linear relationship was observed in the range from $0.33 \mu \mathrm{M}$ to $2.3 \mu \mathrm{M}$. The linear equation was $\mathrm{y}_{2}=0.7522 \mathrm{x}_{2}+0.03665\left(\mathrm{R}^{2}=0.98615\right)$ with a LOD as low as $35 \mathrm{nM}(3 \mathrm{~s}, \mathrm{n}=11)$. Compared with the $1: 1$ binding strategy mentioned above for which the LOD was $1.3 \mu \mathrm{M}$ and $1.0 \mu \mathrm{M}$, respectively, these results clearly demonstrate that the amplification strategy greatly enhances the sensitivity of zeatin and systemin detection, and the LODs were dropped almost two orders of magnitude.

\subsection{Selectivity analysis}

The specificity of this method is investigated by challenging with several analogs. As shown in Fig. 6, the 
fluorescence signals for targets (zeatin and systemin) caused the strongest increase in fluorescence intensity of FAM and ROX. The structure difference between trans-zeatin $(\mathrm{Z})$ and dihydrozeatin (DHZ) is small, and only lies in the $\mathrm{N}^{6}$-isoprenoids substituent. Therefore, dihydrozeatin also showed certain affinity to aptamer in the selection work of DNA aptamers that bind to zeatins. As a consequence, DHZ caused a negligible fluorescence response of ROX and a relatively weak increase in fluorescence intensity of FAM. While The other analogs of zeatin (ATP) and other peptides could not cause significant fluorescence response. Therefore, this proposed method has a satisfactory selectivity.

\subsection{Cross Reaction Analysis}

For simultaneous detection of zeatin and systemin, it is necessary to demonstrate whether there is cross reaction between the Zeatin probe and Systemin probe, as the cross reaction is a crucial analytical parameter regarding the assay specificity and thus the reliability of the multiplex detection. As show in Fig. 7, when there is only zeatin existed in the sample solution, significant fluorescence emission of FAM was observed. In contrast, when the sample contained only systemin, correspondingly, only the fluorescence emission intensity of ROX increased. What's more, when the sample contained systemin with high concentration, only a weak increase in fluorescence intensity of FAM was recorded. These results showed that the cyclic enzymatic amplification method exhibited high tolerance of various targets in multiplexed plant hormones detection to a certain extent.

\subsection{Determination of target in complex matrix}

We further challenged the detection toward the target in relatively complex biological matrix. $20 \%$ plant tissue culture containing a great deal of necessary ions and molecules was chosen in this work. Different concentrations of targets were added into the $20 \%$ plant tissue culture. The recovery of the targets detected in plant tissue culture by the proposed sensing strategy ranged from 91.2 to $121.1 \%$, which were satisfactory for quantitative assays performed in biological samples (Table 1).

\section{Conclusions}

In conclusion, we have demonstrated the first use of the signal amplified aptasensor for simultaneous 
detection of zeatin and systemin with high sensitivity and selectivity. Using this fluorometric method, the detection limits of zeatin and systemin are $60 \mathrm{nM}$ and $35 \mathrm{nM}$, respectively. This sensitivity is about two orders of magnitude higher than that of traditional fluorometric methods without amplification. Furthermore, there is no cross-reaction between these two targets. Therefore, the proposed method may open up a new possibility for plant hormones analysis.

\section{Acknowledgments}

This work was supported by the National Science Foundation of China (91417301, 21275109 and 21205089) and the Science Fund for Creative Research Groups of Hubei province (2014CFA002).

\section{References}

[1] F. Du, G. Ruan, H. Liu, Analytical methods for tracing plant hormones, Anal. Bioanal. Chem., 403(2012) 55-74.

[2] R. Bari, J.D. Jones, Role of plant hormones in plant defence responses, Plant Mol. Biol., 69(2009) 473-88.

[3] C. Nishimura, Y. Ohashi, S. Sato, T. Kato, S. Tabata, C. Ueguchi, Histidine kinase homologs that act as cytokinin receptors possess overlapping functions in the regulation of shoot and root growth in Arabidopsis, The Plant cell, 16(2004) 1365-77.

[4] M. Kojima, T. Kamada-Nobusada, H. Komatsu, K. Takei, T. Kuroha, M. Mizutani, et al., Highly sensitive and high-throughput analysis of plant hormones using MS-probe modification and liquid chromatography-tandem mass spectrometry: an application for hormone profiling in Oryza sativa, Plant Cell Physiol., 50(2009) 1201-14.

[5] M. Ghassemian, E. Nambara, S. Cutler, H. Kawaide, Y. Kamiya, P. McCourt, Regulation of abscisic acid signaling by the ethylene response pathway in Arabidopsis, The Plant cell, 12(2000) 1117-26.

[6] C.D. Rock, X. Sun, Crosstalk between ABA and auxin signaling pathways in roots of Arabidopsis thaliana (L.) Heynh, Planta, 222(2005) 98-106.

[7] T.H.D. Ho, A. Gomez-Cadenas, R. Zentella, J. Casaretto, Crosstalk between gibberellin and abscisic acid in cereal aleurone, J. Plant Growth Regul., 22(2003) 185-94. 
[8] J. Zhang, Y. Li, X. Zhang, B. Yang, Colloidal Self-Assembly Meets Nanofabrication: From TwoDimensional Colloidal Crystals to Nanostructure Arrays, Adv. Mater., 22(2010) 4249-69.

[9] X. Pan, X. Wang, Profiling of plant hormones by mass spectrometry, J. Chromatogr. B: Anal. Technol. Biomed. Life Sci., 877(2009) 2806-13.

[10] P. Tarkowski, L.Y. Ge, J.W.H. Yong, S.N. Tan, Analytical methods for cytokinins, Trac-Trend Anal. Chem., 28(2009) 323-35.

[11] J.H. Fu, X.H. Sun, J.D. Wang, J.F. Chu, C.Y. Yan, Progress in quantitative analysis of plant hormones, Chin. Sci. Bull., 56(2011) 355-66.

[12] Y. Du, B. Li, E. Wang, "Fitting" makes "sensing" simple: label-free detection strategies based on nucleic acid aptamers, Acc. Chem. Res., 46(2013) 203-13.

[13] M. Mascini, I. Palchetti, S. Tombelli, Nucleic acid and peptide aptamers: fundamentals and bioanalytical aspects, Angew. Chem., Int. Ed., 51(2012) 1316-32.

[14] X. Ma, Z. Chen, J. Zhou, W. Weng, O. Zheng, Z. Lin, et al., Aptamer-based portable biosensor for platelet-derived growth factor-BB (PDGF-BB) with personal glucose meter readout, Biosens. Bioelectron., 55(2014) 412-6.

[15] T. Bing, T. Chang, X. Yang, H. Mei, X. Liu, D. Shangguan, G-quadruplex DNA aptamers generated for systemin, Bioorg. Med. Chem., 19(2011) 4211-9.

[16] C. Qi, T. Bing, H. Mei, X. Yang, X. Liu, D. Shangguan, G-quadruplex DNA aptamers for zeatin recognizing, Biosens. Bioelectron., 41(2013) 157-62.

[17] C. Liu, G. Mao, C. Su, X. Ji, Z. Chen, Z. He, Aptamer-functionalized CdTe: $\mathrm{Zn}^{2+}$ quantum dots for the detection of tomato systemin, Anal Methods, (2015).

[18] L. Cui, X. Lin, N. Lin, Y. Song, Z. Zhu, X. Chen, et al., Graphene oxide-protected DNA probes for multiplex microRNA analysis in complex biological samples based on a cyclic enzymatic amplification method, Chem. Commun., 48(2012) 194-6.

[19] Y. Xie, X. Lin, Y. Huang, R. Pan, Z. Zhu, L. Zhou, et al., Highly sensitive and selective detection of miRNA: DNase I-assisted target recycling using DNA probes protected by polydopamine nanospheres, Chem. Commun., 51(2015) 2156-8.

[20] Y. He, G. Huang, H. Cui, Quenching the chemiluminescence of acridinium ester by Graphene oxide for label-free and homogeneous DNA detection, ACS Appl. Mater. Interfaces, (2013).

[21] B. Liu, Z. Sun, X. Zhang, J. Liu, Mechanisms of DNA sensing on Graphene oxide, Anal. Chem., 85(2013) 7987-93.

[22] Y. He, X. Xing, H. Tang, D. Pang, Graphene oxide-based fluorescent biosensor for protein detection via terminal protection of small-molecule-linked DNA, Small, 9(2013) 2097-101.

[23] K.P. Loh, Q. Bao, G. Eda, M. Chhowalla, Graphene oxide as a chemically tunable platform for optical applications, Nat. Chem., 2(2010) 1015-24. 
[24] C.-H. Lu, J. Li, J.-J. Liu, H.-H. Yang, X. Chen, G.-N. Chen, Increasing the sensitivity and single-base mismatch selectivity of the molecular beacon using Graphene oxide as the "Nanoquencher", Chem. - Eur. J., 16(2010) 4889-94.

[25] E. Morales-Narváez, A. Merkoçi, Graphene oxide as an optical biosensing platform, Adv. Mater., 24(2012) 3298-308.

[26] C.H. Lu, H.H. Yang, C.L. Zhu, X. Chen, G.N. Chen, A graphene platform for sensing biomolecules, Angew. Chem., Int. Ed., 48(2009) 4785-7.

[27] N. Varghese, U. Mogera, A. Govindaraj, A. Das, P.K. Maiti, A.K. Sood, et al., Binding of DNA nucleobases and nucleosides with graphene, ChemPhysChem, 10(2009) 206-10.

[28] C.H. Lu, C.L. Zhu, J. Li, J.J. Liu, X. Chen, H.H. Yang, Using graphene to protect DNA from cleavage during cellular delivery, Chem. Commun., 46(2010) 3116-8.

[29] Y. Pu, Z. Zhu, D. Han, H. Liu, J. Liu, J. Liao, et al., Insulin-binding aptamer-conjugated graphene oxide for insulin detection, Analyst, 136(2011) 4138-40.

[30] C.H. Lu, J. Li, M.H. Lin, Y.W. Wang, H.H. Yang, X. Chen, et al., Amplified aptamer-based assay through catalytic recycling of the analyte, Angew. Chem., Int. Ed., 49(2010) 8454-7.

[31] M. Zhang, H.N. Le, B.C. Ye, Graphene Oxide-Based Fluorescent "on/off” Switch for Visual Bioassay Using “Molecular Beacon”-Hosted Hoechst Dyes, ACS Appl. Mater. Interfaces, (2013).

\section{Figure Captions}

Fig. 1. The principle of cyclic enzymatic amplification method for simultaneous detection of zeatin and systemin based on GO protected and quenched aptamers.

Fig. 2. Synchronous scanning fluorescence spectrum for the specificity analysis with (a): GO, Zeatin probe and Systemin probe; (b) GO, Zeatin probe, Systemin probe, zeatin and systemin; (c): GO, Zeatin probe, Systemin probe and DNase I; (d): GO, Zeatin probe, Systemin probe, zeatin, systemin and DNase I. Experimental conditions: GO, $5 \mu \mathrm{g} \mathrm{mL}^{-1}$; Systemin probe, $5.0 \mathrm{nM}$; Zeatin probe, $4.0 \mathrm{nM}$; systemin, $5.0 \mu \mathrm{M}$; zeatin, $5.0 \mu \mathrm{M}$; DNase I, $0.5 \mathrm{U}$.

Fig. 3. (A) Changes in the fluorescence intensity of Zeatin probe $(4.0 \mathrm{nM}$, left) and Systemin probe $(5.0 \mathrm{nM}$, right) upon increasing the concentration of GO, with concentrations of $0,1.0,1.7,2.3,3.7,5.0,6.7,8.3,10(\mu \mathrm{g}$ 
$\mathrm{mL}^{-1}$ ) (from top to bottom). (B) Stern-Volmer plot of Zeatin probe (4.0 nM, black) and Systemin probe (5.0 $\mathrm{nM}$, red) quenched by GO in buffer solutions.

Fig. 4. (A) The synchronous scanning fluorescence spectrum for different concentrations of zeatin: 5, 10, 15, $20,25,30,35,40,50 \mu \mathrm{M}$ (from bottom to top) and different concentrations of systemin: 3.3, 5.0, 6.7, 8.3, 10, $11.7,13.3,20,30 \mu \mathrm{M}$ (from bottom to top). The $\left(F-F_{0}\right) / F_{0}$ (fluorescence change) vs the concentrations of zeatin (B) and systemin (C). The inset shows the linear relationship between the $\left(F-F_{0}\right) / F_{0}$ and the concentrations of zeatin(B) and systemin(C), respectively. Experimental conditions: Systemin probe, $5.0 \mathrm{nM}$; Zeatin probe, $4.0 \mathrm{nM}$; GO, $5.0 \mu \mathrm{g} / \mathrm{mL}$.

Fig. 5. (A) The synchronous scanning fluorescence spectrum for different concentrations of zeatin: 0.67, 1.0, $1.3,1.7,2.0,2.3,3.0,4.0,6.0 \mu \mathrm{M}$ (from bottom to top) and different concentrations of systemin: $0.33,0.67$, $1.0,1.3,1.7,2.0,2.3,3.0,5.0 \mu \mathrm{M}$ (from bottom to top), The $\left(F-F_{0}\right) / F_{0}$ (fluorescence change) vs the concentrations of zeatin (B) and systemin (C). The inset shows the linear relationship between the $\left(F-F_{0}\right) / F_{0}$ and the concentrations of the targets: zeatin (B) and systemin (C), respectively. Experimental conditions: Systemin probe, $5.0 \mathrm{nM}$; Zeatin probe, $4.0 \mathrm{nM}$; GO, $5.0 \mu \mathrm{g} / \mathrm{mL}$; DNase I, $0.5 \mathrm{U}$.

Fig. 6. The $\left(F-F_{0}\right) / F_{0}$ (fluorescence change) histogram of the sensing system including $5.0 \mu \mathrm{M}$ of zeatin and systemin $(\mathrm{Z}+\mathrm{S})$, dihydrozeatin (DHZ), adenosine triphosphate (ATP), Peptide 1(P 1) and Peptide 2 (P 2).

Fig. 7. The synchronous scanning fluorescence spectrum for the specificity analysis with: (A) Different concentrations of zeatin: $0,0.67,1.3,2.0 \mu \mathrm{M}$ (from bottom to top). (B) Different concentrations of systemin: 0 , $0.33,1.0,1.7 \mu \mathrm{M}$ (from bottom to top). 
Table 1. Determination of the targets in $20 \%$ plant tissue culture with this amplification strategy.

\begin{tabular}{|c|c|c|c|c|c|c|}
\hline \multirow{2}{*}{$\begin{array}{l}\text { Sample } \\
\text { number }\end{array}$} & \multicolumn{2}{|c|}{$\begin{array}{c}\text { Added } \\
(\mu \mathrm{M})\end{array}$} & \multicolumn{2}{|c|}{$\begin{array}{c}\text { Found } \\
(\mu \mathrm{M})\end{array}$} & \multicolumn{2}{|c|}{$\begin{array}{c}\text { Recovery } \\
\%\end{array}$} \\
\hline & zeatin & systemin & zeatin & systemin & zeatin & systemin \\
\hline 1 & 1.00 & - & 0.912 & - & 91.2 & - \\
\hline 2 & - & 1.00 & - & 1.120 & - & 112.0 \\
\hline 3 & 1.00 & 0.50 & 0.973 & 0.492 & 97.3 & 98.4 \\
\hline 4 & 1.00 & 1.00 & 1.211 & 1.075 & 121.1 & 107.5 \\
\hline 5 & 2.00 & 1.00 & 2.163 & 1.051 & 108.2 & 105.1 \\
\hline
\end{tabular}




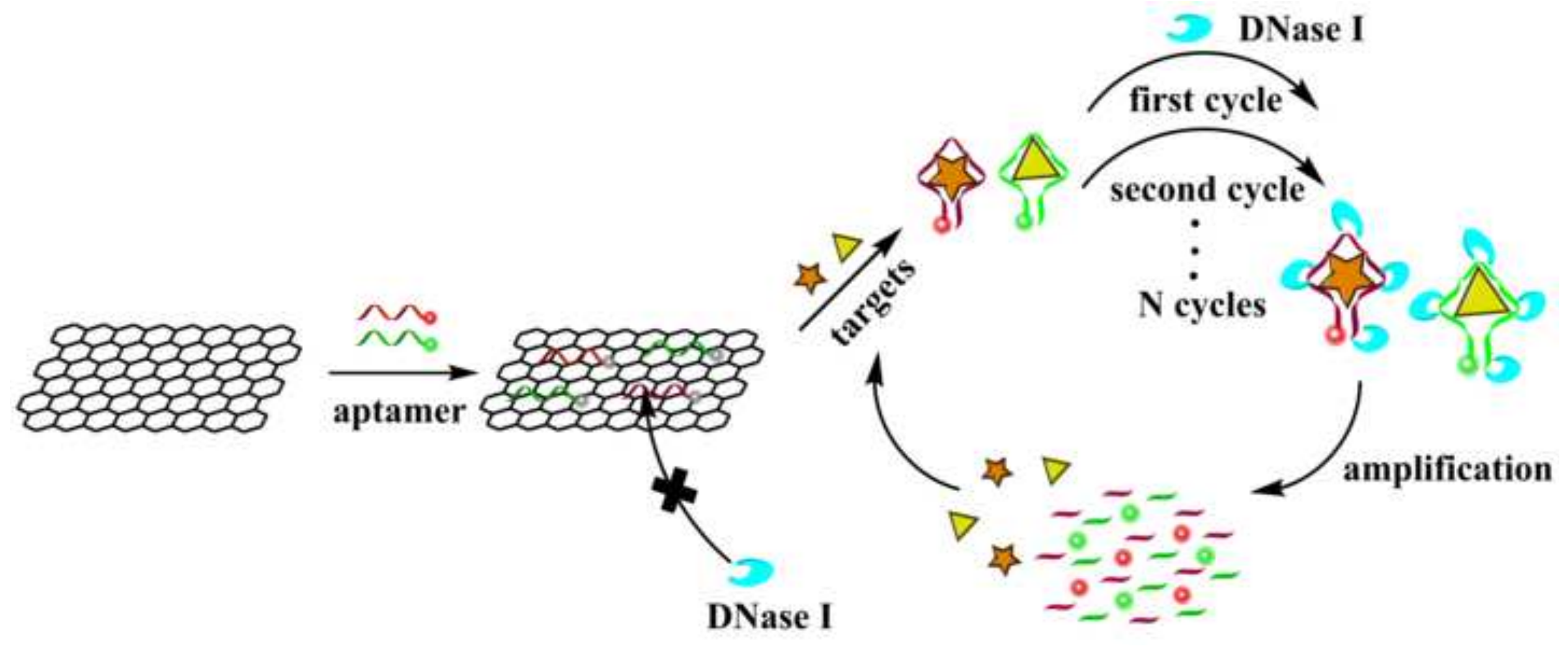




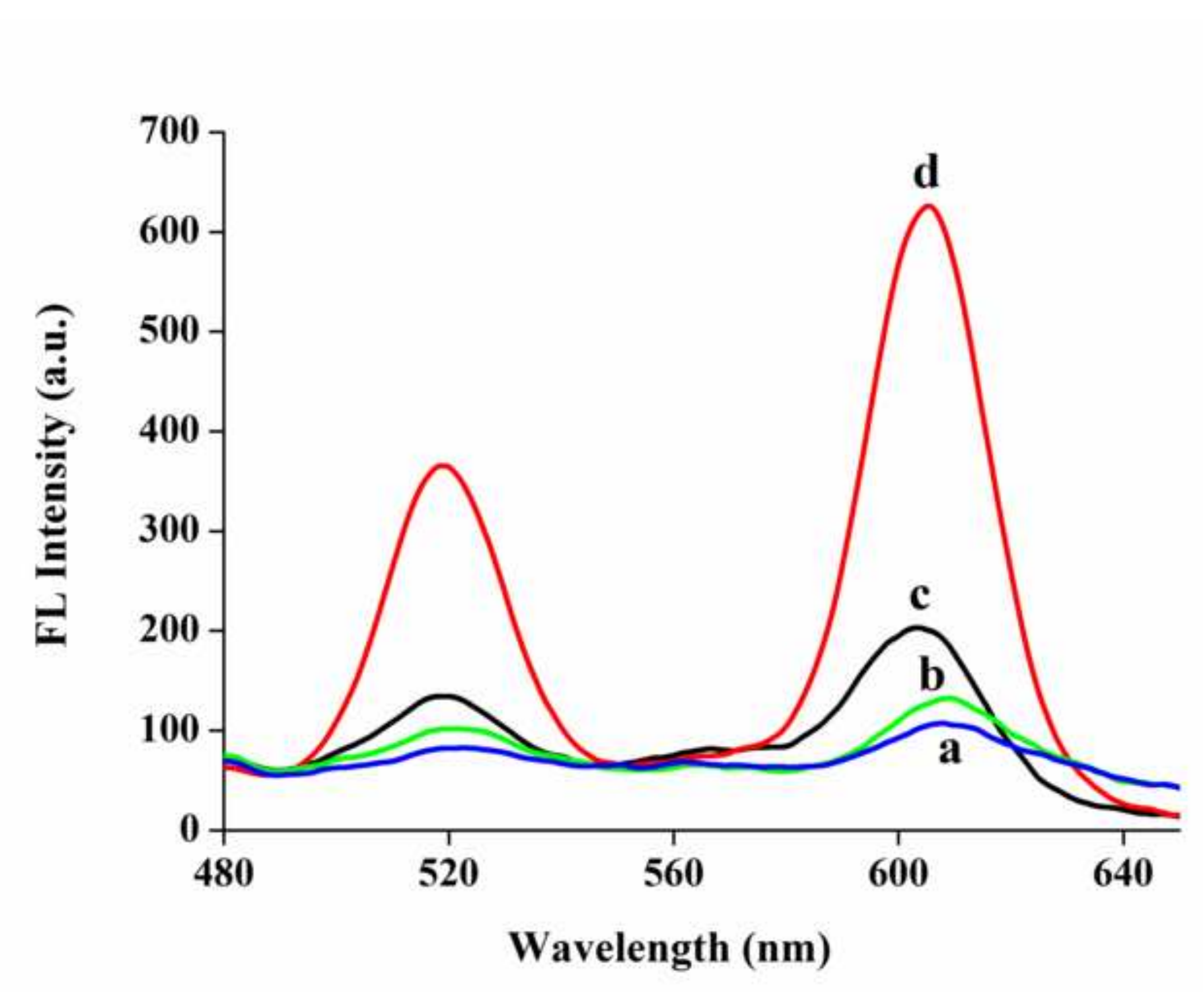

.
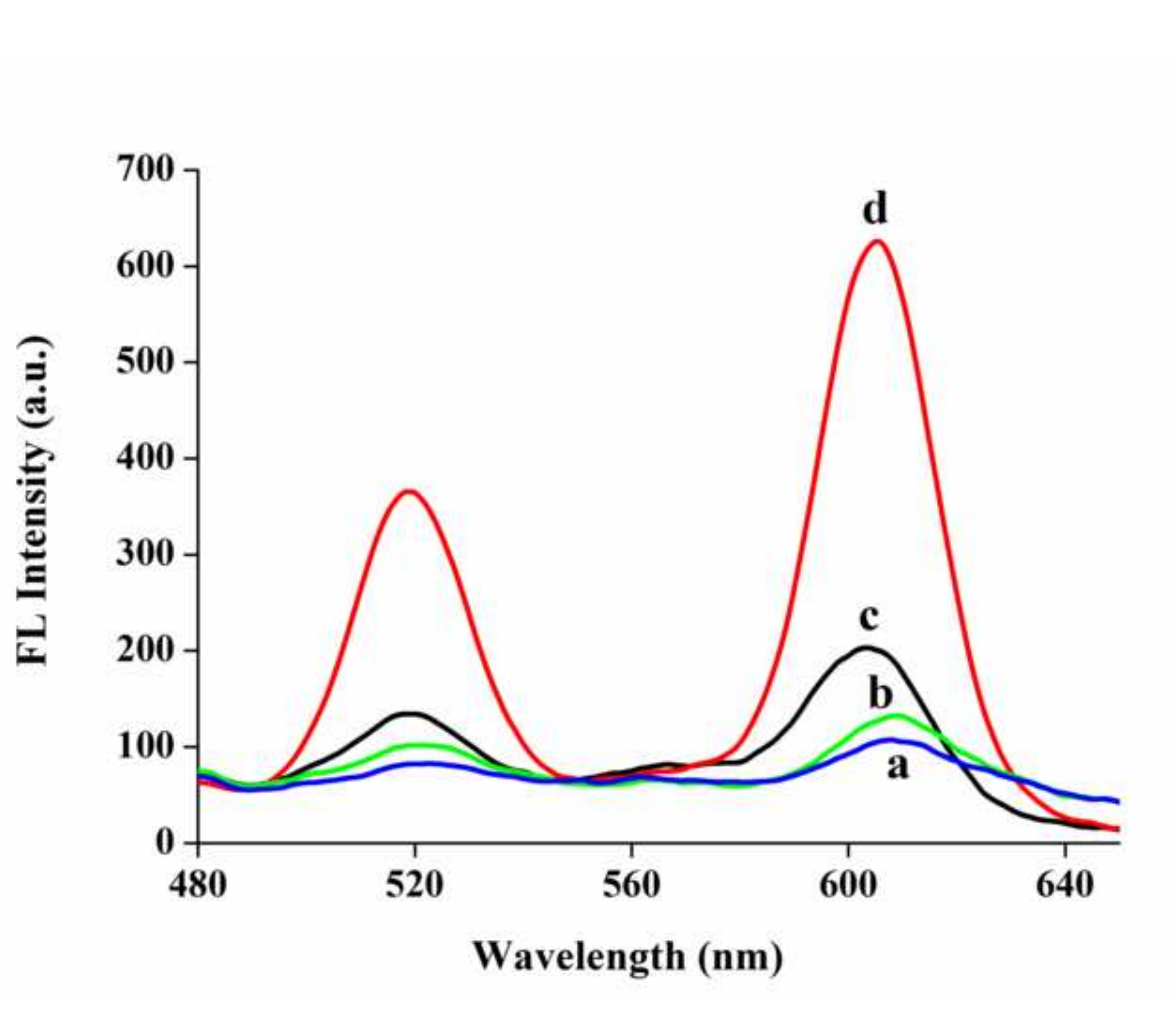

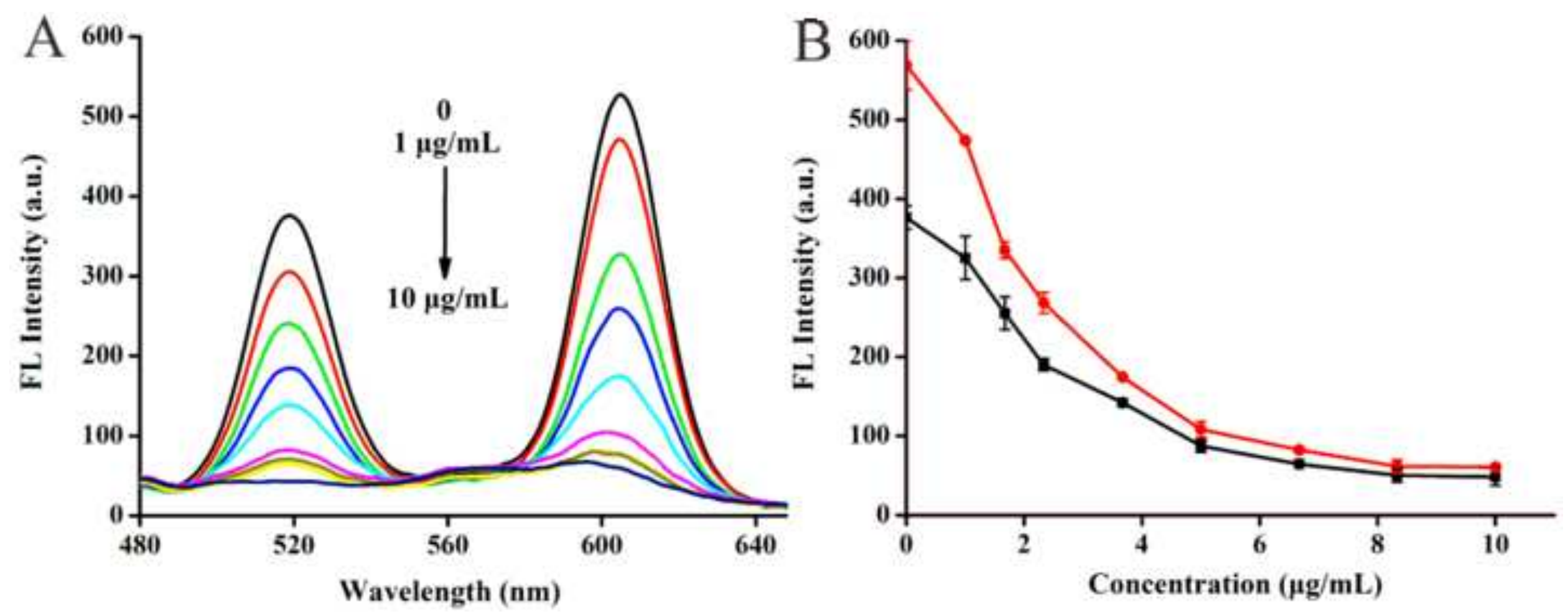

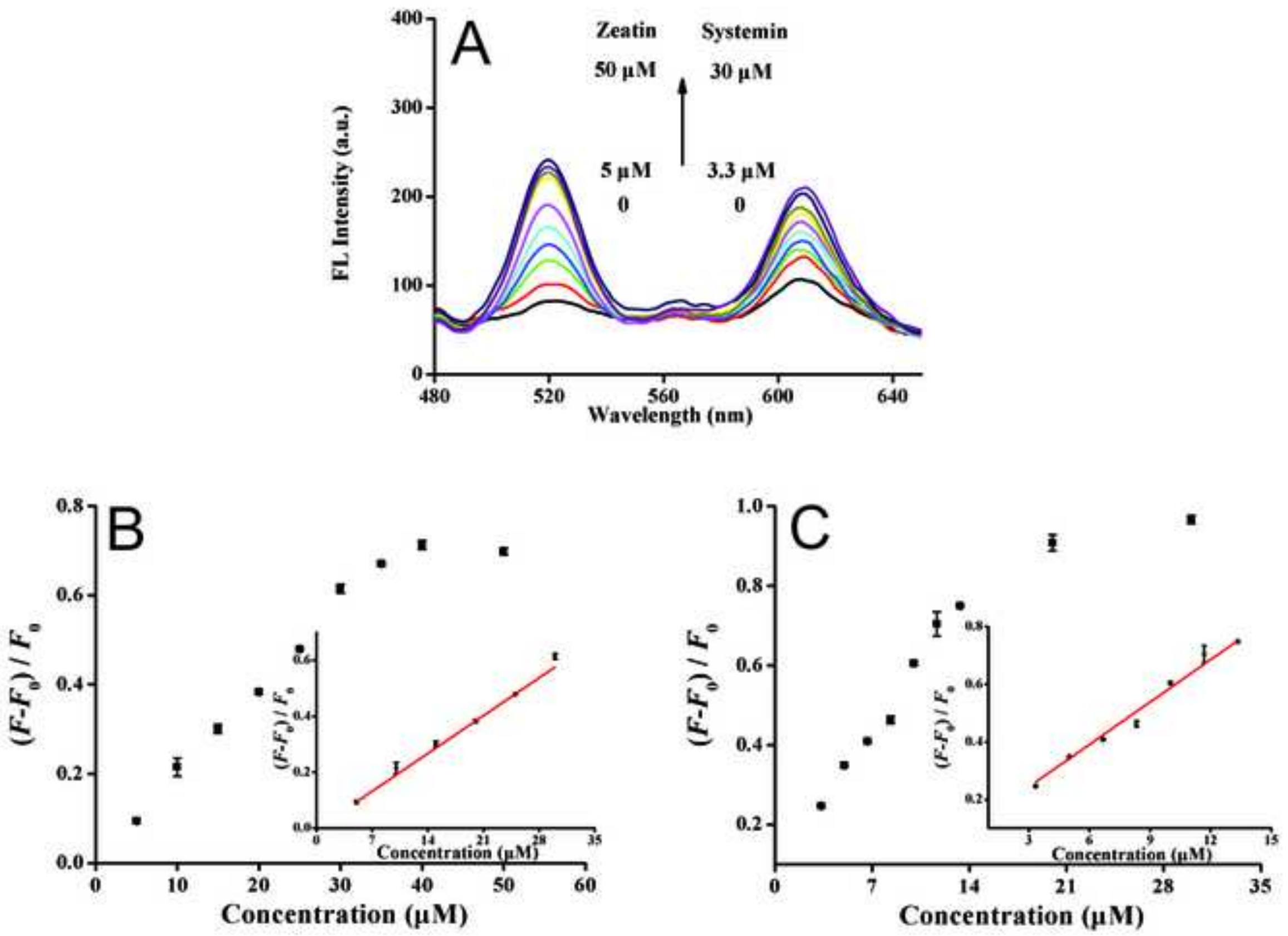

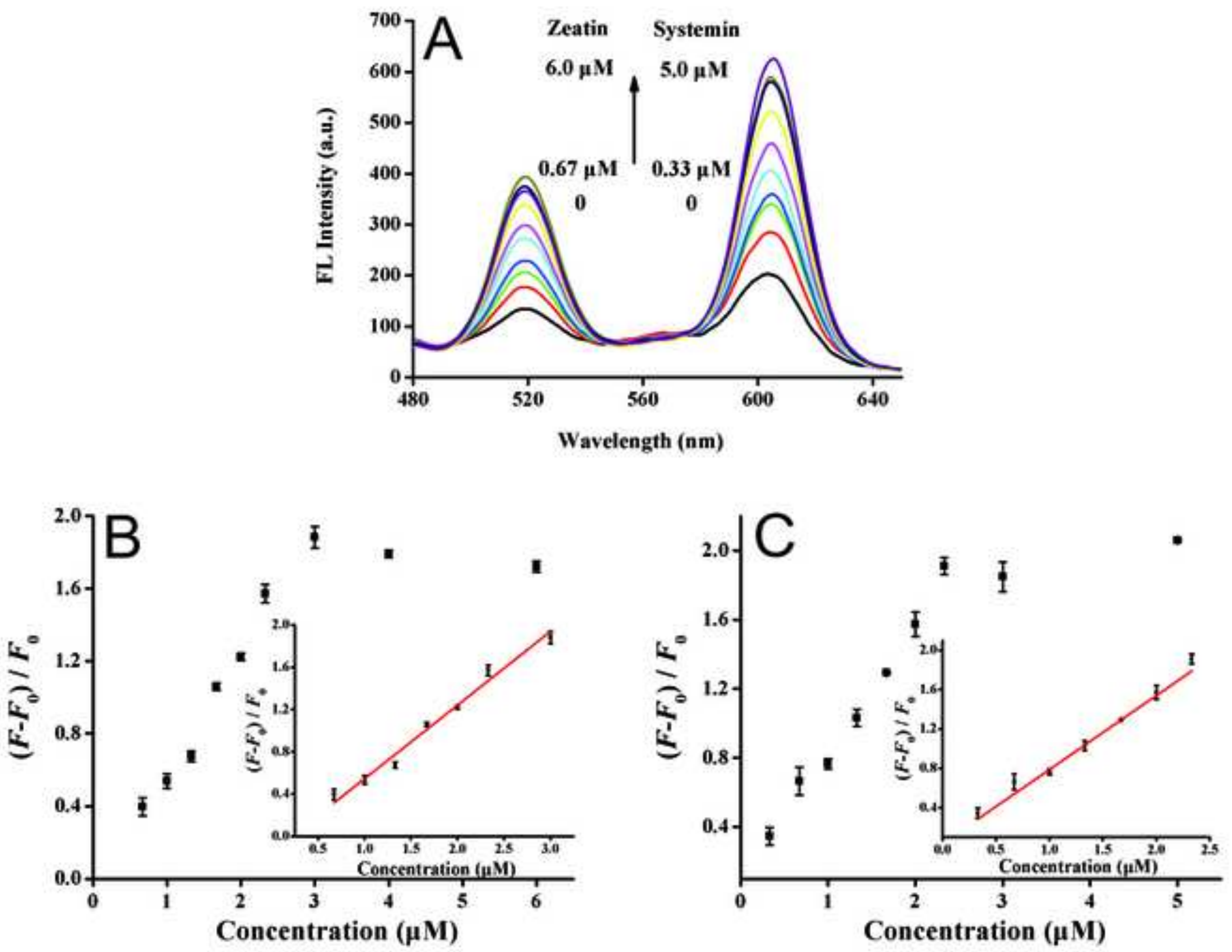

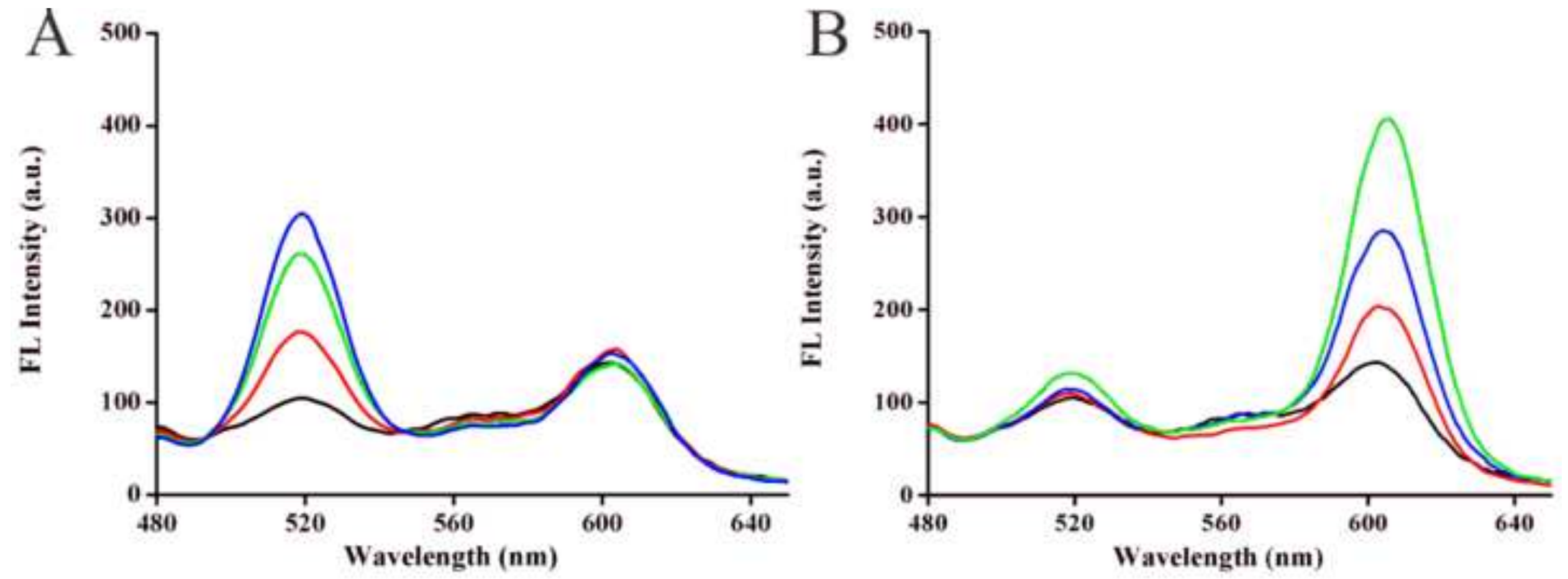


\section{Biographies}

Chen Su is currently a master degree student in Wuhan University. Her research interest is biosensor based on DNA molecular light switches.

Chen Liu is currently a master degree student in Wuhan University. Her current research interests include the development of novel fluorescent nano materials and fluorescent sensors.

Jinyang Chen is currently a Ph.D. student in Wuhan University. He works on the development of novel techniques for biosensing in microfluidic systems.

Zilin Chen is a Professor of School of Pharmaceutical Sciences at Wuhan University. He received his Ph.D. degree in Applied Chemistry from Tokyo Metropolitan University in 2000. His research interest focuses on the pharmaceutical analysis.

Zhike He is a Professor of Analytical Chemistry at Wuhan University. He received his Ph.D. degree in Analytical Chemistry from Wuhan University in 1995. His research interest covers Nano-bio-analytical chemistry and application for biology and medicine. 